through TRUGENE HIV-1 Genotyping assay. Phylogenetic analyses were performed by the maximum likelihood method with MEGA software.

Results Among the 101 sequences analysed, 54 (53.5\%) were HIV-1 subtype B and 47 (46.5\%), non-B subtypes. The recent infection rate was $22.2 \%(n=12)$ and $19.1 \%(n=09)$ for subtypes $\mathrm{B}$ and non-B. In non-B subtypes there were a significant decrease in CD4+ $\mathrm{T}$ cells count between recent and long-term infections compared to subtype $B(p=0.002)$. There was no statistical difference in viral load levels and infection status for the analysed subtypes.

Conclusion Decreases in CD4 $+\mathrm{T}$ cells count in the course of infection by non-B subtypes may indicate a propensity for disease progression by these variants. Thus, genotyping, antiretroviral resistance, and infection status assessments are important for monitoring local epidemics.

\section{P3.123 POPULATION GROWTH AND EVOLUTIONARY HISTORY OF HIV-1 B AND F SUBTYPES IN THE NORTHEAST BRAZIL}

${ }^{1}$ Kledoaldo Oliveira de Lima, 2 Élcio Leal, ${ }^{3}$ Ana Maria Salustiano Cavalcanti, ${ }^{3}$ Daniela Medeiros Salustiano, ${ }^{4}$ Heloísa Ramos Lacerda. ${ }^{1}$ Post-graduation in Tropical Medicine, Federal University of Pernambuco, Recife, PE, Brazil; ${ }^{2}$ Hospital das Clínicas, Federal University of Pernambuco, Recife, $P E$, Brazil; ${ }^{3}$ Institute of Biotechnology, Federal University of Pará, Belém, PA, Brazil; " Sector of Virology, Central Laboratory of Public Health, Recife, $P E$, Brazil

\subsection{6/sextrans-2017-053264.358}

Introduction Despite low prevalence in the world, the HIV-1 subtype $\mathrm{F}$ is expanding in the state of Pernambuco, Northeast - Brazil, being the most prevalent subtype after subtype B. Increase of the frequency of other recombinants $\mathrm{BF}$ in the region, denoting the high capacity of recombination of subtype F with B. Thus, the objective of this work was to characterise the transmission dynamics between subtypes $B$ and $F$ in the state of Pernambuco, Northeast - Brazil.

Methods One hundred and fifty-six sequences of HIV-1 infected individuals were obtained from five Voluntary and Counselling Testing Centres (VCTs) in the state of Pernambuco (Northeast - Brazil), of which 103 were HIV-1 B and 53, HIV-1 F. Samples were collected between 2002 and 2009. We used the Bayesian Markov chain Monte Carlo (BMCMC) coalescent framework to estimate the ancestral genealogy, phylodynamics and evolutionary parameters such as nucleotide substitution rates per year and time to the most common ancestor (tMRCA) with BEAST package version 1.8.1.

Results Pattern of population growth are similar between subtype B in Brazil and in Pernambuco showing a pattern of steep exponential growth followed by a plateau in the diversity. Population dynamics of subtype F shows a moderate growth phase continuously expanding and the beginning of infection started later than that of global subtype $F$ infection. The coalescent method also provided the date of introduction of HIV-1 in Pernambuco since the inferred time to the most recent common ancestral (tMRCA) was 1978 (95\% CI: 19711981) and 1982 (95\% CI: 1977-1986) respectively for the subtypes B and F.

Conclusion Introduction of subtype B occurred earlier in other regions of Brazil than in Pernambuco (Northeast, Brazil). Subtype $\mathrm{F}$ is in population expansion around the world and in Brazil. In addition, introduction of subtype $\mathrm{F}$ in Pernambuco (Northeast) was later than in the country (1982, CI95\%:
1977-1986). These findings support the hypothesis that the viral variability of HIV-1 is increasing in Brazil with the spread of subtypes non-B.

\section{P3.124 ADOLESCENTS LIVING WITH HIVIAIDS - DIFFERENT APPROACHES IN DIFFERENT MODES OF HIV TRANSMISSION}

${ }^{1}$ Krysna Pires, ${ }^{2}$ Eveline Xavier-Souza, ${ }^{2}$ Maiara Timbó, ${ }^{1}$ Raiza Trindade, ${ }^{1}$ Camila Souza, ${ }^{3}$ Ana Gabriela Travassos. 'Universidade do Estado da Bahia, Salvador - BA, Brazil; ${ }^{2}$ Universidade Federal da Bahia, Salvador - BA, Brazil; ${ }^{3}$ Centro Estadual Especializado em Diagnóstico, Assistência E Pesquisa (CEDAP), Salvador - BA, Brazil

\subsection{6/sextrans-2017-053264.359}

Introduction Recently, the number of adolescents infected with HIV has been increasing worldwide, but there is still little information available on the characteristics of this population. The aim of this study is to compare the characteristics of adolescents living with HIV attending a Sexually Transmitted Infection (STI) Referral Centre according to the modes of transmission.

Methods A cross-sectional study evaluating adolescents between 10 and 19 years of age, carried out from January to August 2012, at the state STI reference centre in Bahia, Brazil. Sociodemographic and clinical data were obtained by reviewing charts and analysed through SPSS 20.0.

Results A total of 123 adolescents living with HIV were attended during the study period, $76.3 \%$ (90/118) of them acquired the virus through vertical transmission (VT), whereas $22.9 \%(27 / 118)$ acquired via sexual transmission (ST). Regarding the adolescents with VT, the group was younger than the ST, with $93.7 \%<16$ years old $(\mathrm{p}<0.01$, OR $20.35,95 \% \mathrm{CI}$ 6.70-61.83); 78.9\% attended school $(\mathrm{p}<0.01$, OR 0.15 , 95\% CI $0.04-0.61), 62.1 \%$ did not work $(p=0.02), 55.2 \%$ denied use of alcohol ( $\mathrm{p}<0.01$, OR 0.07 , 95\% CI $0.01-0.65)$, and $98.7 \%$ also denied sexual debut $(\mathrm{p}<0.01$, OR $<0.01$, 95\% CI 0.00-0.03). On the other hand, patients with a ST infection started their sexual life earlier, with a mean age of $12.9( \pm 3.74)$ years, $93.8 \%$ of them had had previous gestation ( $\mathrm{p}<0.01$, OR 27.5, 95\% CI 2.89-262.3), 75.0\% had HPV co-infection $(\mathrm{p}<0.01$, OR 11.5 , 95\% CI 2.03-64.78) and $88.9 \%$ had another STI in the period $(p<0.01$, OR $45.71,95 \%$ CI 5.30-394.42). The VT group showed rates of $93,5 \%$ of AIDS diagnosis $(\mathrm{p}<0.01$, OR 72 ., 95\% CI 16.41$315.98)$ and $100 \%(p=0.01)$ had had opportunistic infections; $93,1 \%$ performed genotyping $(\mathrm{p}=0.02$, OR $5.87,95 \% \mathrm{CI}$ $1.27-27.09)$ and $85.4 \%$ used ART $(\mathrm{p}<0.01$, OR 8.54 , 95\% CI 2.72-26.85).

Conclusion We observed two distinct groups, defined according to the modes of transmission, both showing specific characteristics. Thus, is it evident the need of customise the health care and promotion according to each group.

\section{P3.125 KNOWLEDGE ON SEXUALLY TRANSMITTED INFECTIONS/ HIV, SEXUAL RISK BEHAVIOURS AND UTILISATION OF DROP-IN-CENTRES AMONG KEY AFFECTED POPULATION IN MYANMAR}

${ }^{1}$ Kyaw-Min Htut, ${ }^{1}$ Myo-Myo Mon, ${ }^{2}$ Ye Aung. 'Ministry of Health And Sports, Yangon, Myanmar Burma; ${ }^{2}$ Save The Children, Yangon, Myanmar Burma

10.1136/sextrans-2017-053264.360 
Introduction In Myanmar, People Who Inject Drugs (PWIDs) has the highest HIV prevalence of $18.7 \%$ in comparing to other key population. Establishment of drop-in-centre (DIC) and provision of services has been proved as successful intervention for key population. An assessment was conducted to determine the knowledge on sexually transmitted infections (STI)/HIV and utilisation of drop-in-centres among drug users after the establishment of DIC and out-reach activities.

Methods A community-based, post-intervention assessment was conducted using quantitative and qualitative methods in underserved areas of north-western border in Myanmar.

Results A total of 202 drug users, 156 PWIDs and 46 People Who Use Drugs (PWUDs), were included in the study. Their age ranged from ranged from 18 to 60 years with the mean age of $34.9 \pm 8.2$ years. Regarding STI knowledge, no one sought to treat STD at the beginning of the intervention but $4 \%$ of drug users searched for the treatment at the end. Over $25 \%$ knew at least one symptom of STI in men while only $15.3 \%$ recognised at least one symptom in women. Knowledge on higher risk of blood borne infections such as Hepatitis B $(25 \%$ vs. $51 \%, \mathrm{p}<0.1)$, Hepatitis C $(8 \%$ vs. $13 \%$, $\mathrm{p}<0.05)$ was improved in comparing to pre-intervention. HIV testing rate within six months was also increased from $10 \%$ to $74 \%(\mathrm{p}<0.001)$. Regarding sexual behaviours, percent of drug users who had sex with paid sex partner and who had more than one sexual partner within 6 months were significantly reduced $(11 \%$ vs. $0.5 \%, \mathrm{p}<0.05 ; 11 \%$ vs. $1 \%$, $\mathrm{p}<0.05)$. Regarding drop in centre (DIC), more drug users were aware of DIC $(54 \%$ vs. $91 \%, \mathrm{p}<0.01)$ and ever visited there $(22 \%$ vs. $76 \%, p<0.01)$. Almost all of them $(94 \%)$ ever received DIC services consisted of HIV testing and counselling $(47 \%)$ and health education service (68\%). Nearly half of them (46\%) received outreach services and mobile HIV testing covered about $16 \%$.

Conclusion Improvement in knowledge and behaviour regarding STI/HIV and DIC utilisation were seen among the drug users after the intervention.

\section{P3.126 COUNTY-LEVEL SOCIODEMOGRAPHIC FACTORS ASSOCIATED WITH REPORTED CONGENITAL SYPHILIS IN UNITED STATES 2012-2015}

Kyle Bernstein, Kendra Cuffe, Sarah Kidd, Virginia Bowen, Elizabeth Torrone, Jami Leichliter. CDC - Atlanta, USA

\subsection{6/sextrans-2017-053264.361}

Introduction Pregnant women - In the US, Congenital Syphilis (CS) rates have increased 48\% between 2012 and 2015. We explored county level sociodemographic factors associated with having reported at least one CS case during 2012 and 2015 among all US counties.

Methods The $321(10.2 \%)$ US counties that reported at least 1 CS case during 2012-2015 were compared to the remaining $2829(89.8 \%)$ US counties with no reported CS. Multivariable logistic regression models were constructed to assess factors independently associated with counties reporting CS using adjusted Odds Ratios (aOR). County level factors examined included: $\%$ population black [above or below the national median],\% Hispanic population [above or below the national median], Medicaid expansion state, violent crime rate per 100,000 , and the $\%$ change in adult male and female syphilis cases between during 2012 and 2015. Final models were determined by comparing likelihood ratio statistics.

Results Independent county level factors associated with reporting at least one CS case during 2012-2015 were: Medicaid expansion state $[\mathrm{aOR}=2.24,95 \%$ CI $1.51-3.34], \%$ black population $[\mathrm{aOR}=2.86,95 \%$ CI $1.79-4.57], \%$ Hispanic $[\mathrm{aOR}=2.13,95 \% \mathrm{CI} 1.41-3.24], 10$ unit change in violent crime rate $[\mathrm{aOR}=1.04,95 \% \mathrm{CI} 1.02-1.05]$, and 10 unit change in $\%$ change in female syphilis $[\mathrm{aOR}=1.02,95 \% \mathrm{CI}$ 1.00-1.03].

Conclusion CS in the US is highly geographically focused with only $10.2 \%$ of counties reporting any cases in the past 4 years. Socioeconomic and demographic factors working at the community level are associated with increased odds of having reported a case of CS. Interventions to prevent CS in the US should focus at community, as well as individual level.

\section{P3.127 ANALYSIS ON ACCURATE DIAGNOSIS AND STANDARDISED TREATMENT OF REPORTED SYPHILIS CASES IN SHENZHEN}

Lan Li-Na, Wu Xiao-Bing, Zhang Chun-Lai, Wen Li-Zhang, Tang Fen, Hong Fu-Chang. Shenzhen Centre for Chronic Disease Control, Shenzhen - China Popular Republic

\subsection{6/sextrans-2017-053264.362}

Introduction To investigate the accurate rate of diagnosis and the standardised rate of treatment of reported syphilis cases in Shenzhen and to provide evidence for further prevention strategies.

Methods Data of syphilis cases reported from July 1, 2013 to June 30, 2014 were downloaded from the 'China Information System for Disease Control and Prevertion'. Randomised sampling method was used to select cases from the download database. Medical staff compared the information from the selected cases with doctors' daily record, patients' laboratory test results and pharmacy prescriptions from medical institutions. The accuracy of diagnosis and standardisation of treatment were evaluated based on the Diagnostic Criteria and Management of Syphilis Guidelines (Ministry of Health, China, 2007).

Results This study totally selected 1589 syphilis cases for evaluation. The accurate rate of diagnosis was $95.53 \%$ for total, and the rates were $83.59 \%, 96.60 \%, 97.73 \%, 100.00 \%$ and $97.37 \%$ respectively for primary, secondary, tertiary, congenital and latent syphilis. Nearly $66.08 \%$ cases were treated with penicillin therapy and $1.76 \%$ cases were treated with replacement therapy. The standardised rate of treatment was $67.84 \%$. Conclusion The accurate rate of syphilis diagnosis is high in Shenzhen, however, the standardised rate of treatment is far from the goal set in the national syphilis control plan. It suggests that training and supervision should be enhanced and modification of communicable disease card (such as adding the diagnosis and treatment information of syphilis cases) should be considered, to improve the rate of accurate diagnosis and standardised treatment of syphilis cases. 